Article

\title{
Planar Dual Polarized Metasurface Array for Microwave Energy Harvesting
}

\author{
Maged A. Aldhaeebi ${ }^{1}$ and Thamer S. Almoneef ${ }^{2, *}$ \\ 1 Department of Electronics and Communication Engineering, Hadhramout University, \\ Mukalla 50512-50511, Yemen; maged.aldhaeebi@gmail.com \\ 2 Electrical Engineering Department, College of Engineering, Prince Sattam Bin Abdulaziz University, \\ Al-Kharj 11942, Saudi Arabia \\ * Correspondence: t.almoneef@psau.edu.sa
}

Received: 26 October 2020; Accepted: 18 November 2020; Published: 24 November 2020

check for updates

\begin{abstract}
The design of a dual polarized metasurface collector based on the metamaterial full absorption concept for electromagnetic energy harvesting is introduced. Unlike previous metamaterial absorber designs, here the power absorbed is mostly dissipated across a resistive load rather than within the dielectric substrate. This is achieved by channeling the absorbed power to an optimal resistive load through a via hole. The simulation results show that a power absorption efficiency of $98 \%$ is achieved at an operating frequency of $2 \mathrm{GHz}$ for a single unit cell. A super unit cell consisting of four cells with alternating vias was also designed to produce a dual polarized metasurface. The simulation results yielded a radiation to AC efficiency of around $98 \%$ for each polarization.
\end{abstract}

Keywords: planar meatsurface; dual polarized absorbers; energy harvesting; rectenna

\section{Introduction}

The microwave energy harvesting system (MEHS) has recently received much attention in the field of developing rectenna arrays based on metamaterials [1]. The two main components of any microwave energy harvesting system are an antenna and a rectification circuit. The antenna is used for collecting incident electromagnetic radiated power and converting it to AC (Radiation to AC efficiency), whereas a rectification circuit is used for converting the collected AC power to DC (AC to DC efficiency) [2]. To enhance the overall performance of the MEHS, the antenna part is the essential part that should be improved for capturing and converting the incident microwave energy into AC power.

In the MEHS, the polarization and the direction of an incident electromagnetic wave are considered to be unknown, which, if they differ, can reduce the overall performance of the system due to the loss of the receiving polarization mismatch power [3,4]. In order to enhance the overall performance of the MEHS in terms of the total efficiency (Radiation to DC efficiency), the polarization of the antenna is to be considered as one of the fundamental factors for designing or synthesising the Wireless Power Transfer (WPT) system.

In the literature, conventional dual polarized single antennas integrated with the rectification circuit are used for energy harvesting system [3-9]. However, the conventional dual polarized single antennas capture and collect relatively small amounts of RF power. In order to increase the amount of the available ambient power and convert it to AC, recent studies have focused on developing highly efficient harvesting systems based on metasurface arrays [10-15].

Several studies have concentrated on developing dual polarized rectenna arrays based on designating dual polarized metasurface antenna arrays [14-23]. However, the introduced studies in [14-19] developed dual polarized metasurface arrays based on a dual layer configuration with 
multiple vias which adds complicity to the fabrication process and adds cost to the overall energy harvesting system.

In this study, a novel design of a dual polarized rectenna array made of an ensemble of metasurface unit cells for higher radiation to AC efficiency is introduced. The proposed metasurface array has the capability of collecting electromagnetic energy over a wide impedance bandwidth, allowing for incorporating a rectification circuit without the use of a matching network. In addition, the proposed design avoids the use of vias, utilizing a planar single layer to host the collectors which reduces the design cost and makes the design much simpler to configure.

\section{Design Methodology, Analyses and Simulation Results of the $2 \times 2$ Supercell}

A unit cell of the proposed dual polarized metasurface harvester is shown in Figure 1a, which consists of a loop with an embedded dipole in the middle. The dipole contains three extended small parallel arms forming three gaps. A supercell, which contains 4 unit cells, is designed on top of a Rogers RO4003C dielectric substrate having a $\tan \delta=0.0027$ and a dielectric constant of $\epsilon_{r}=3.38$ with a thickness of $1.524 \mathrm{~mm}$. The cell dimensions of the unit cell is designed at an operating frequency of $2 \mathrm{GHz}$ with $I=20 \mathrm{~mm}, g=0.5 \mathrm{~mm}, r=3.5 \mathrm{~mm}, z=3.5 \mathrm{~mm}, w=7 \mathrm{~mm}$ and copper thickness $=35 \mu \mathrm{m}$. The loop comprises two identical gaps of $\mathrm{c}=0.5 \mathrm{~mm}$. The electromagnetic energy is received and concentrated within the feed gap of the dipole having a gap length of $g=0.5 \mathrm{~mm}$. A terminated variable resistive load is placed across the feed gap to simulate the ability of the metasurface unit cell to receive and consume the collected energy. A ground plane reflector is allocated at an optimum stand off distance of $S=15 \mathrm{~mm}$ away from the proposed unit cell as shown in Figure 1a. The unit cell was simulated using CST Microwave Studio [24]. The proposed dual polarized supercell consists of identical $2 \times 2$ unit cells as shown in Figure 1b, where the separation between adjacent cells is sep. The dimensions of the proposed dual polarized super unit cell is designed such that it operates at $2 \mathrm{GHz}$ with a $=40 \mathrm{~mm}$. Figure 2 shows the numerical results $S_{11}$ of the dual polarized planar $2 \times 2$ super unit cell.

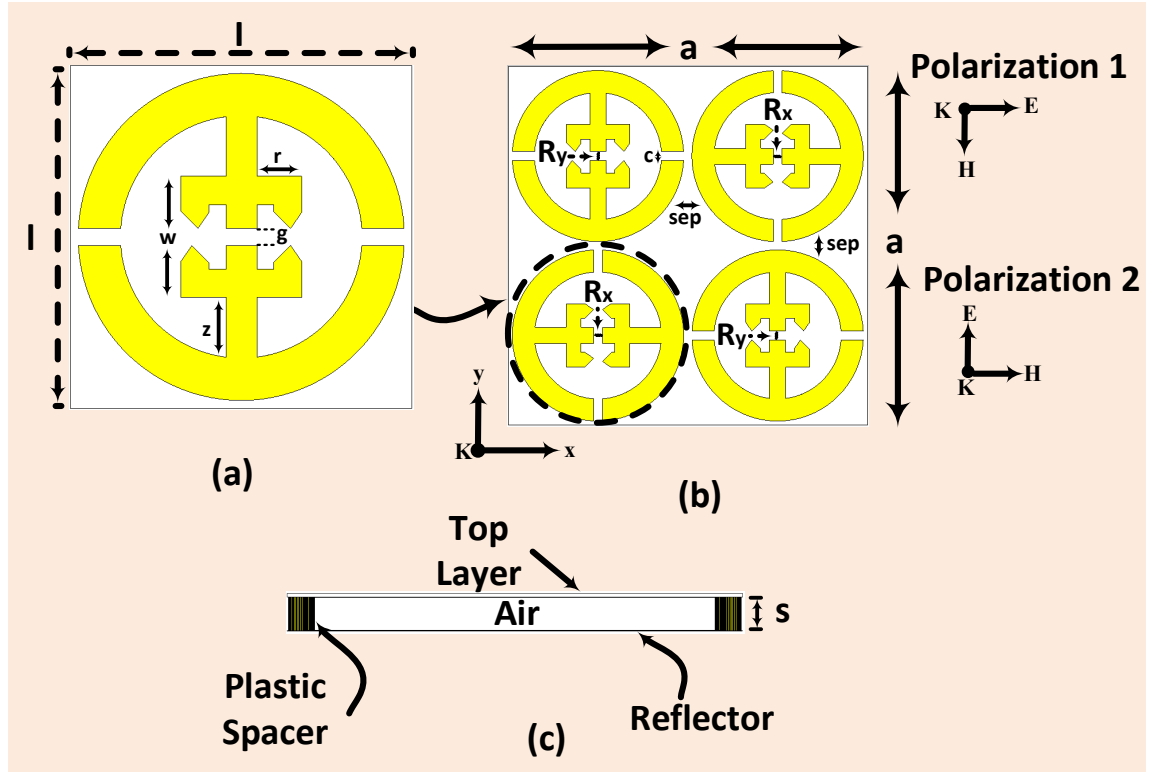

Figure 1. Geometry of the proposed dual polarized planar metasurface for a: (a) unit cell, (b) $2 \times 2$ super unit cell (c) top layer view of the dual polarized supercell. 


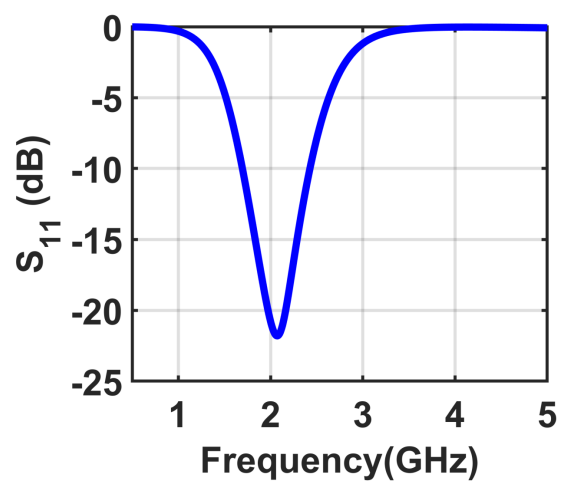

Figure 2. Numerical results of the dual polarized planar $2 \times 2$ supercell demonstrating the $S_{11}$.

The supercell is proposed to be $2 \times 2$ to capture the incident power for both polarizations ( $\mathrm{X}$ and $\mathrm{Y}$ ) of the incident power. The proposed dual polarized supercell consists of $2 \times 2$ unit cells for capturing both polarizations of the incident wave as shown in Figure $1 b$, where the responsibility of the top right and bottom left cells are for capturing polarization 1 of the incident wave as illustrated in the figure, and the top left and bottom right cells are utilized for capturing polarization 2. Note that the cell is able to capture the available electromagnetic energy when the middle dipole is parallel to the incident electric field. When the incident wave is x-polarized (polarization 1: $\theta=0^{\circ}$ ) at the operating frequency of $2 \mathrm{GHz}$, the incident power is delivered to the resistive loads (Rx) along $\mathrm{x}$ of top right and bottom left cells; however, the power is almost zero across the resistive loads (Ry) along y of top left and bottom right cells. On the other hand, when the incident wave is y-polarized (polarization 2: $\theta=90^{\circ}$ ) at the operating frequency, the incident power is developed across the resistive loads (Ry) along y of top left and bottom right cells while the incident power is almost zero across the resistive loads ( $\mathrm{xx}$ ) along $\mathrm{x}$ of top right cell and bottom left cell.

In the simulation setup, first, the distance off between the proposed supercell and the ground plane reflector was varied from $S=5 \mathrm{~mm}$ to $S=20 \mathrm{~mm}$. In all four cases of separation distance values (S), the resistive loads are swept over the range from $R=50 \Omega$ to $R=300 \Omega$. Then the efficiency of the middle supercell containing a $2 \times 2$ sub unit cells is computed. Note that the reason of using the middle supercell to calculate the efficiency is to ensure that the supercell experience infinite periodicity. When using a finite array, one expects that the input impedance of the outer most cells to be slightly different from the input impedance of the unit cells located in the middle of the finite array. Such differences in the input impedance are due to the high coupling between the cells experienced at the center of the finite array. The cells located in the peripheral of the finite array experience less coupling than the cells located in the middle of the surface. Therefore, to mimic a periodic infinite array, a supercell containing four cells of both polarizations located in the middle of the finite metasurface is considered. Numerical results of the efficiency of the proposed metasurface with various terminated loads ranging from $R=50 \Omega$ to $R=300 \Omega$ and with separation values of $S=5 \mathrm{~mm}, S=10 \mathrm{~mm}$, $S=15 \mathrm{~mm}$, and $S=20 \mathrm{~mm}$ are showing in Figure 3a-d, respectively. The efficiency peaks when all cells are terminated by a load resistance of $50 \Omega$ with a maximum of $98 \%$ at the separation distance (S) value of $S=15 \mathrm{~mm}$. Furthermore, the metasurface has the capability to capture electromagnetic waves with a wide range of terminated load resistances in the range from $50 \Omega$ to $300 \Omega$ with efficiencies exceeding $85 \%$ as shown in Figure 3c. Because the input impedance of most diodes are within this resistance range, a diode can be mounted right across the metasurface feed gap without a matching network. Thus, the metasurface design can be simplified and the total size of the harvester can be reduced. 


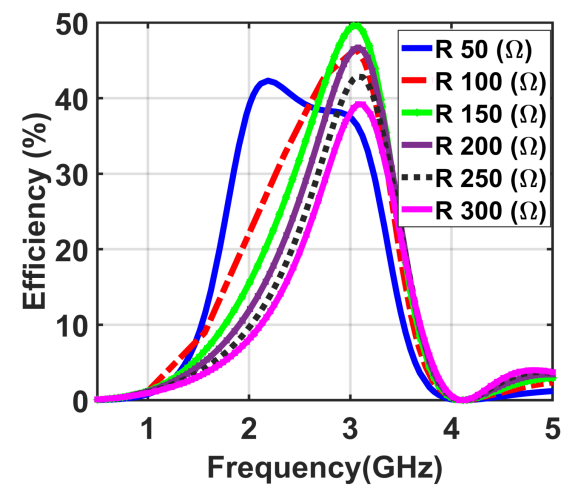

(a):S=5 mm

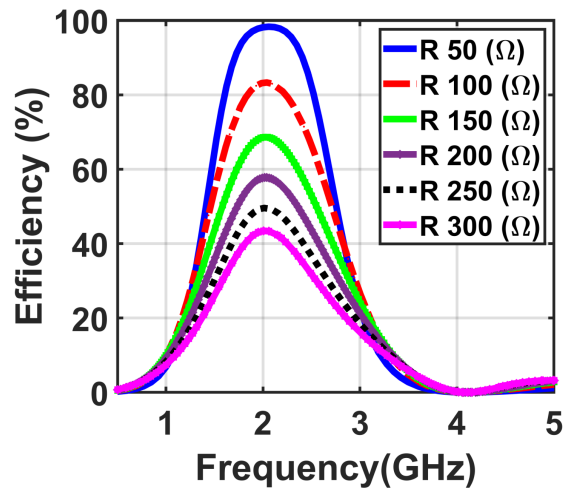

(c): $\mathrm{S}=15 \mathrm{~mm}$

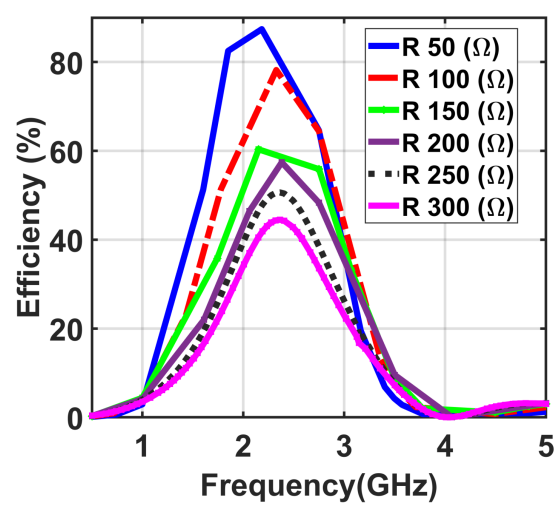

(b): $\mathrm{S}=\mathbf{1 0} \mathrm{mm}$

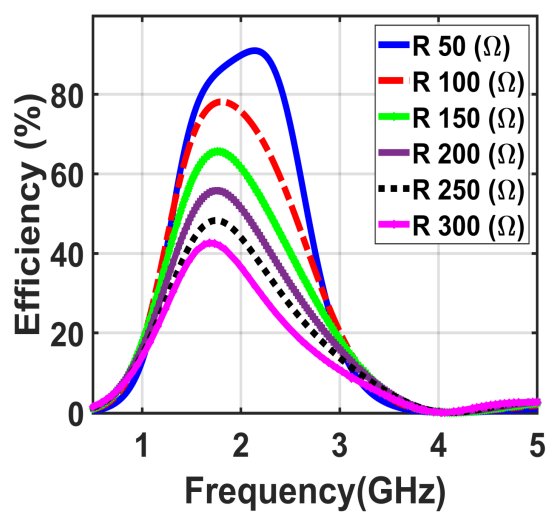

(d): $S=20 \mathrm{~mm}$

Figure 3. Numerical results showing the efficiency of the proposed antenna with various terminated loads ranging from $50 \Omega$ to $300 \Omega$, and having a separation distance of (a) $S=5 \mathrm{~mm}$, (b) $S=10 \mathrm{~mm}$, (c) $\mathrm{S}=15 \mathrm{~mm}$, and (d) $\mathrm{S}=20 \mathrm{~mm}$.

Next the simulation was extended to show another important factor of the proposed unit cell structure which contributes to the overall efficiency of the proposed $2 \times 2$ supercell, including the gap of the unit cell and the separation between adjacent cells of the proposed $2 \times 2$ super unit cell. To demonstrate this, a numerical simulation study is carried out with different sizes of the gap from $g_{1}=0.2 \mathrm{~mm}$ to $g_{4}=0.5 \mathrm{~mm}$ at the optimum resistive load of $50 \Omega$ and the optimum distance of $S=15 \mathrm{~mm}$ between the surface and the reflector. At all four different dimensions of the gap, the efficiency is recorded over a range of frequencies. Figure $4 \mathrm{a}$ shows the numerical results of the efficiency with varied gap values from $g_{1}=0.2 \mathrm{~mm}$ to $g_{4}=0.5 \mathrm{~mm}$, showing a peak efficiency at the optimum value of gap size of $g=0.5 \mathrm{~mm}$.

The next simulation of the proposed supercell is conducted to illustrate the effect of the lateral separation between adjacent cells of the $2 \times 2$ super unit cell on the overall efficiency of the metasurface. In the simulation study, the separation between adjacent cells is varied between $\operatorname{sep}_{1}=0.3 \mathrm{~mm}$ to $\operatorname{sep}_{6}=1.2 \mathrm{~mm}$. From the results presented in Figure $4 \mathrm{~b}$, the highest efficiency is achieved at the optimum value of the lateral separation length, sep $=0.3 \mathrm{~mm}$. 


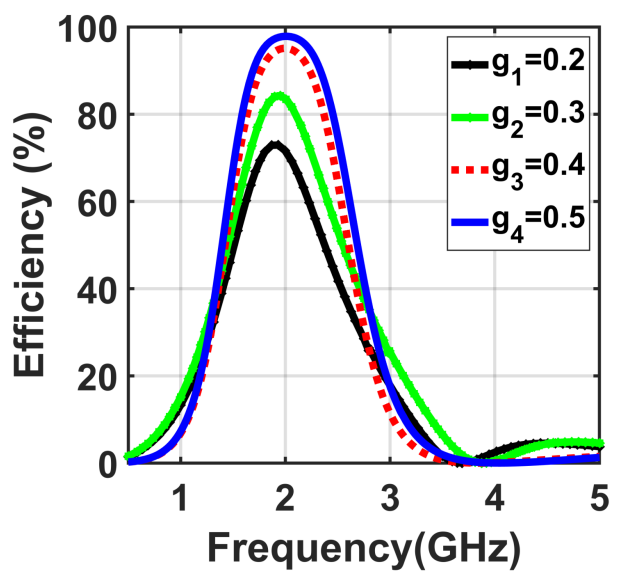

(a)

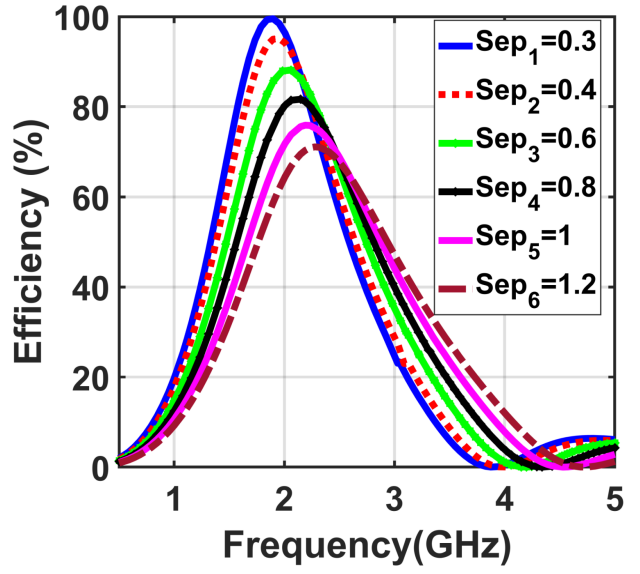

(b)

Figure 4. Numerical results of the presented dual polarized planar $2 \times 2$ supercell demonstrating, (a) the efficiency with varied gaps from $g_{1}=0.2 \mathrm{~mm}$ to $g_{4}=0.5 \mathrm{~mm}$ and (b) the efficiency with various lateral separation distance between adjacent cells.

Next, the proposed $2 \times 2$ super unit cell with the optimal design parameters obtained above is simulated and analyzed in the receiving mode for both normal incident polarizations as illustrated in Figure 1. The numerical result of the radiation to AC efficiency of the supercell of both polarizations is shown in Figure 5, resulting in a radiation to AC conversion efficiency for both polarizations of $98 \%$ at $1.7 \mathrm{GHz}$.

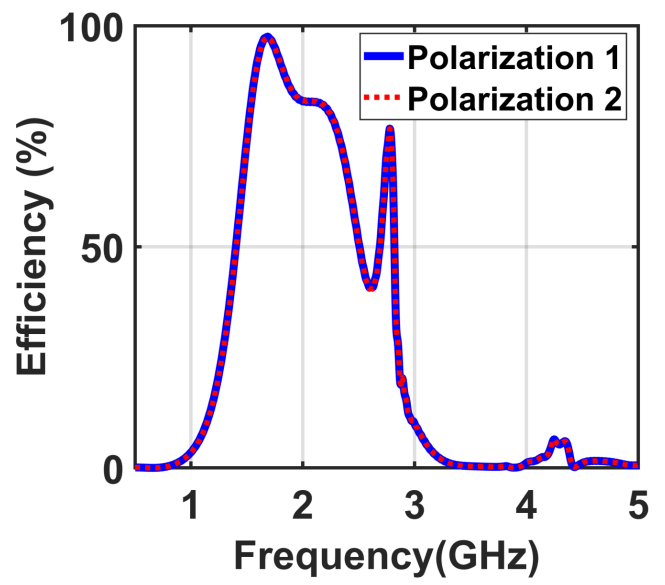

Figure 5. Numerical results of the presented dual polarized planar $2 \times 2$ supercell, showing the radiation to AC efficiency of the supercell for both polarizations.

In order to demonstrate the dual polarized response of the proposed $2 \times 2$ super unit cell, the E-field and surface current distribution were simulated for both polarizations at the resonance frequency. Figure 6 shows the numerical results of the magnitude of the E-field over the surface of the $2 \times 2$ supercell for both polarizations. From the plot, the E-field of each polarization is illustrated across the surface of the $2 \times 2$ supercell. The dominant E-field distributions are active as observed at the upper-left and the bottom-right unit cells of the incident wave at polarization one (TE-mode), while the dominant E-field distributions is amplified within the top right and bottom left unit cells of the incident wave in polarization two (TM-mode). 


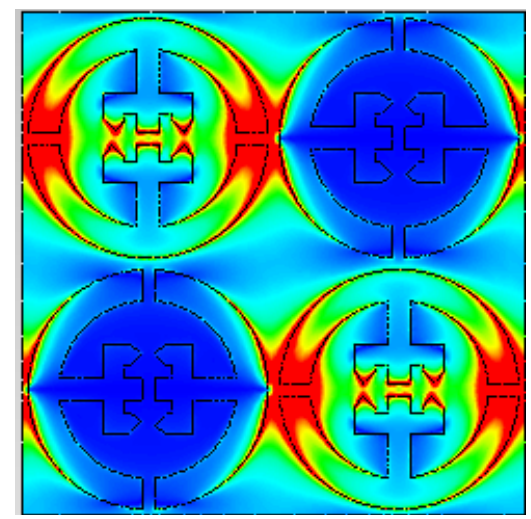

(a)

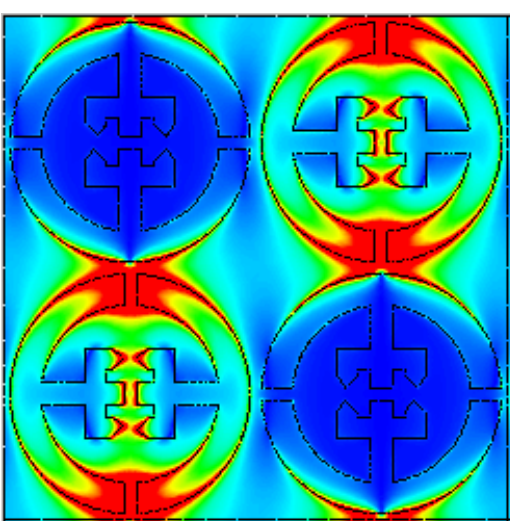

(b)

Figure 6. Numerical results showing the magnitude of the E-field across the surface of the $2 \times 2$ supercell for (a) polarization 1, and (b) polarization 2.

Figure 7 shows the magnitude of the surface current across the surface of the $2 \times 2$ supercell for both polarizations. The current plot illustrates that the current is concentrated at two cells simultaneously for each polarization, depending on the polarization of the incident E-field. From both the E-field and current plots, maximum energy is concentrated at the gap where the resistive loads are located since the absorbed energy is channeled and consumed across the load resistors.

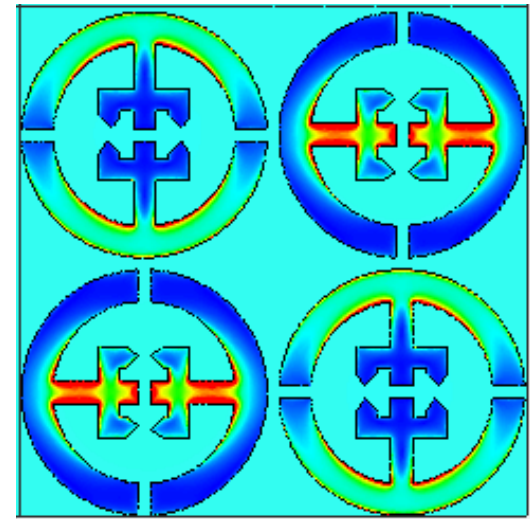

(a)

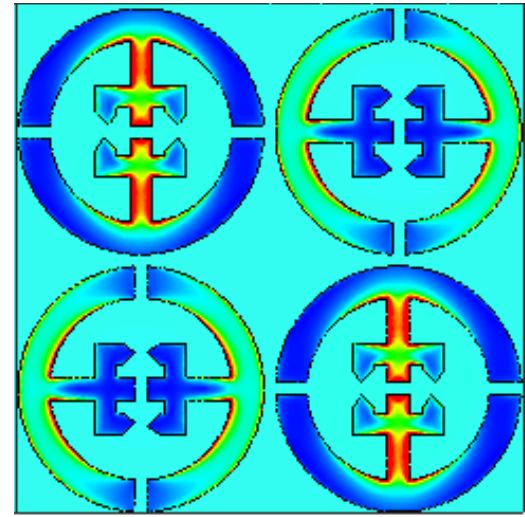

(b)

Figure 7. Numerical results showing the magnitude of the surface current across the surface of the $2 \times 2$ supercell for (a) polarization 1 , and (b) polarization 2 .

The novelty of the proposed supercell is demonstrated by using the three small extended arms in the main middle dipole of the unit cell. The main reason of adding these three extended small arms is to offer the freedom of the design to miniaturize the proposed metasurface. To validate this concept, a simulation was carried out with four cases as the following: (a) the main proposed supercell with the three extended arms where the load resistance is placed in the middle arm, (b) the main proposed supercell without the three extended arms where the load resistance is placed across the middle arm, (c) the main proposed supercell with the three extended arms where the load resistance is placed 
across the right arm, and (d) the main proposed supercell with the three extended arms where the load resistance is placed across the left arm as shown in Figure 8.

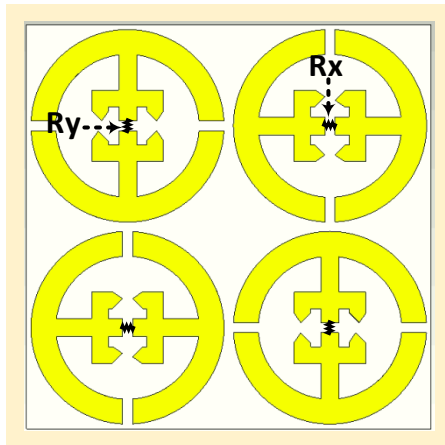

(a)

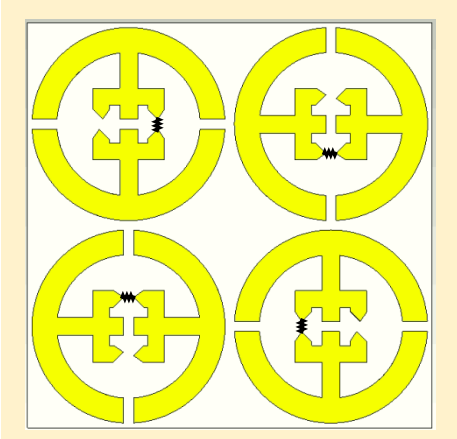

(c)

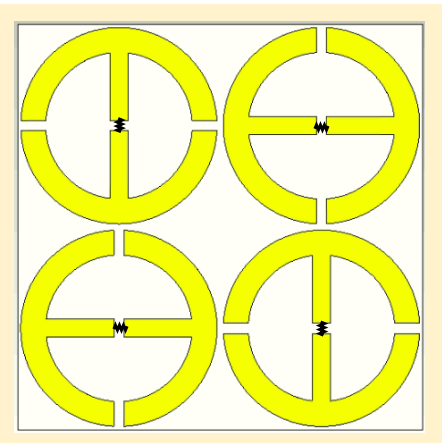

(b)

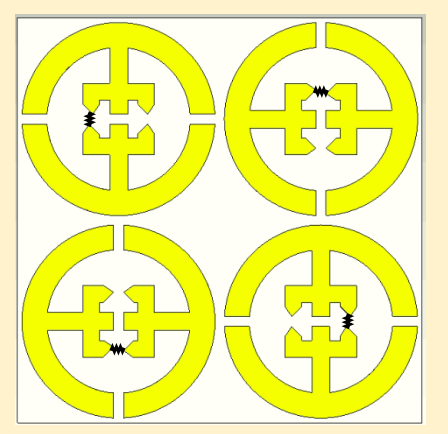

(d)

Figure 8. An illustration showing the four cases of the proposed dual polarized planar supercell: (a) the main proposed supercell with three extended arms where the load resistance is placed in the middle arm, (b) the main proposed supercell without three extended arms where the load resistance is placed in the middle arm, (c) the main proposed supercell without three extended arms where the load resistance is placed in the right arm, and (d) the main proposed supercell without three extended arms where the load resistance is placed in the left arm.

The four cases were simulated and the results are plotted in Figure 9. When comparing cases (a) and (b), it is noticeable that the curves of the efficiency as a function of frequency of both cases are approximately the same, with a maximum efficiency of $98 \%$ at the operating frequency of $2 \mathrm{GHz}$ and the optimum load resistance of $50 \Omega$. However, for cases (c) and (d), we compared the proposed supercell with the three extended small arms, where the load resistance is placed at the right and the left arms as shown in Figure 8c,d respectively. Simulation results show that the efficiency of both cases resulted in a frequency that is red shifted by around $300 \mathrm{MHz}$ which miniaturized the overall electrical size of the harvester while the efficiency is maintained at a maximum of $98 \%$ at the operating frequency of $1.7 \mathrm{GHz}$ as showing in Figure 9. 


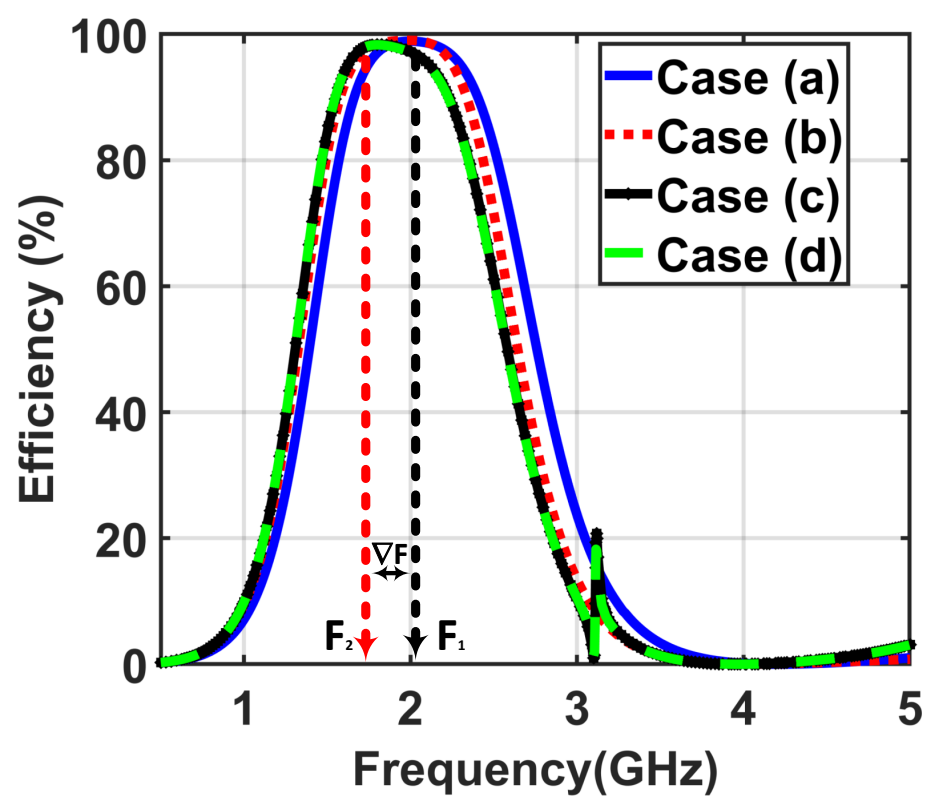

Figure 9. Simulation results of four cases of the proposed supercell: (a) case a (b) case b (c) case c, and (d) case $\mathrm{d}$ as described within the manuscript.

\section{Design Methodology, Analyses and Simulation Results of the $8 \times 8$ Planar Metasurface}

The proposed metasurface harvester consists of a periodic array of identical $8 \times 8$ unit cells with dimensions as explained above and is shown in Figure 10. The overall array size was $\mathrm{L}=210 \mathrm{~mm} \times$ $\mathrm{W}=210 \mathrm{~mm}$. In the numerical simulation, all unit cells were terminated by variable resistive loads, placed across the feed gap of each unit cell. The resistive load mimics a diode that can be later replaced by the load resistor to measure and analyze the radiation to DC efficiency of the metasurface and how it behaves to various input impedances at the feed point. The proposed metasurface was simulated in the receiving mode with a planewave excitation having polarization as illustrated in Figure 10. The efficiency of the metasurface is computed by: $\eta=\frac{P_{d}}{P_{i n}}$, where the input power, $P_{i n}$, is the real power available on the footprint of the harvester in Watts. Note here that $P_{\text {in }}$ can be obtained by multiplying the footprint area of the metasurface by the poynting vector above the surface of the harvester. The developed power, $P_{d}$, can be calculated by summing all the developed powers across all the load resistors of each unit cell. Such power can be either AC or DC depending on the type of harvester and the existence of diodes.

The middle supercell in the $8 \times 8$ array was tested numerically for various incident angles $\theta$ measured with a clockwise rotation of the E field vector as shown in Figure 10. The angle $(\theta)$ was varied with different values from $30^{\circ}$ to $90^{\circ}$ in increments of $30^{\circ}$ as illustrated in Figure 10b. Figure 11 shows the results of the efficiency for different angles $(\theta)$ for a range of frequency sweep points. From the results, regardless of the incident angle value, the efficiency remains the same with the same frequency response. This proposes that the metasurface harvester can collect or receive the electromagnetic energy from the incident planewave equally regardless of the orientation of the parallel $E$ field vector. This efficiency values are calculated by taking the ratio of the total energy dissipated across all the 8 resistors of the middle supercell to the incident power. It is interesting to note here that $98 \%$ of the incident power is consumed across the resistive loads which are responsible for dissipating the energy for each polarization. 


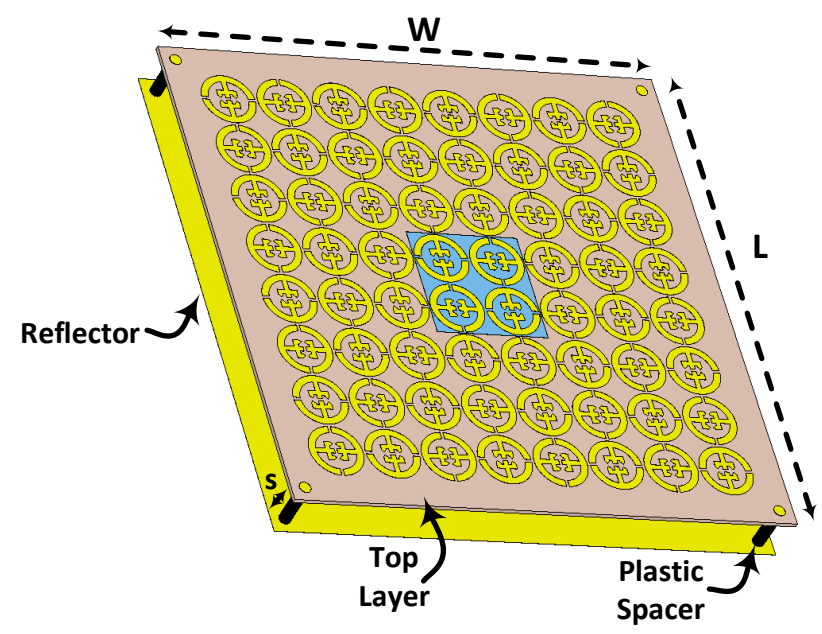

(a)
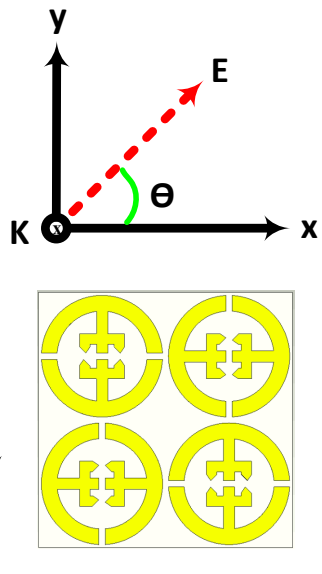

(b)

Figure 10. Geometry of the proposed dual polarized planar metasurface: (a) a perspective view of the proposed dual polarized planar $8 \times 8$ metasurface and (b) a $2 \times 2$ super unit cell with vector representation showing the different incident polarization angles of E-field.

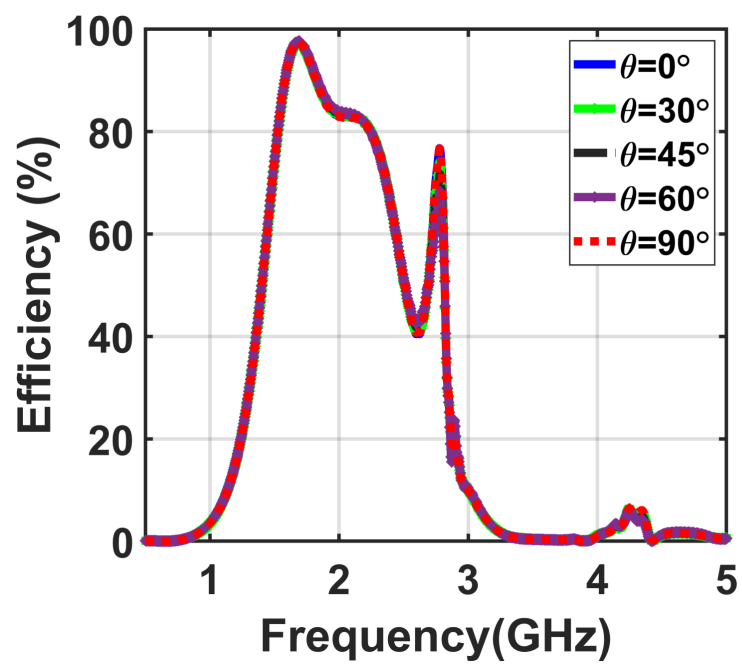

Figure 11. Numerical results of the $8 \times 8$ dual polarized planar metasurface, demonstrating the radiation to AC conversion efficiency as a function of frequency for various incident angles.

The efficiency of the supercell in the $8 \times 8$ proposed metasurface is then analysed as a function of frequency. In this simulation setup, the optimal values of both the spacer and the load resistance were fixed as obtained above. In addition to the two incident polarizations (polarization 1: $\theta=0^{\circ}$ and polarization 2: y-polarized $\theta=90^{\circ}$ ), three additional incident angles of $\theta=30^{\circ}, 45^{\circ}$ and $60^{\circ}$ were also considered as shown in Figure $10 \mathrm{~b}$.

When the incident wave is x-polarized (polarization 1: $\theta=0^{\circ}$ ) at the operating frequency of $2 \mathrm{GHz}$, the incident power is delivered across the resistive load ( $\mathrm{Rx}$ ) along $\mathrm{x}$; however, the incident power is almost zero across the resistive load (Ry) along $\mathrm{y}$. On the other hand, when the incident wave is y-polarized (polarization 2: $\theta=90^{\circ}$ ) at the operating frequency, the incident power is delivered across the resistive load (Ry) along y while the incident power is almost zero across the resistive load $(\mathrm{Rx})$ along $\mathrm{x}$. For various oblique angles $\left(\theta=30^{\circ}, 45^{\circ}\right.$ and $\left.60^{\circ}\right)$ of the incident E-field (please refer to Figure 10b), the harvested power is developed across both the Rx and Ry loads with proportions depending on the angle of the E-field. However, the total captured energy will always remain the 
same since the aggregated total absorbed power is identical regardless of the E-field angle rotation due to the orientation of the middle dipoles of the four cells being orthogonal to each dipole within an adjacent cell.

\section{Analyses and Simulation Results of Radiation to DC Efficiency of the $8 \times 8$ Planar Metasurface}

A rectification circuit mainly consists of a Schottky diode along with a smoothing DC filter which allows passing the DC signal after rectification and rejecting the remaining AC signal. An SMS 3922 Schottky diode was selected for its low series resistance of $9 \Omega$, high breakdown voltage of $20 \mathrm{~V}$, and high switching capability at the operating frequency due to a low junction capacitance of $0.7 \mathrm{pF}$. The rectifier's AC to DC efficiency was analysed using the diode model presented in [25]. From the diode model, the efficiency of the rectification circuit as a function of the diode and load resistance is plotted and shown in Figure 12. The diode can be placed across the feed of the harvester and replace the $50 \Omega$ optimal resistor found in the previous analyses. To avoid any reflections between the harvester and the diode, the diode along with the rectifier should have an input impedance of $50 \Omega$. From the plot, the AC to DC efficiency when the diode resistance is $50 \Omega$ is approximatively $60 \%$. Therefore, the overall Radiation to DC efficiency can be obtained by taking the product of the two sub systems, the harvester (Radiation to AC) and the rectifier (AC to DC) as: $\eta=\left(\eta_{\text {rad }}\right.$ to AC $) \times$ $\left(\eta_{A C}\right.$ to $\left.D C\right)$.

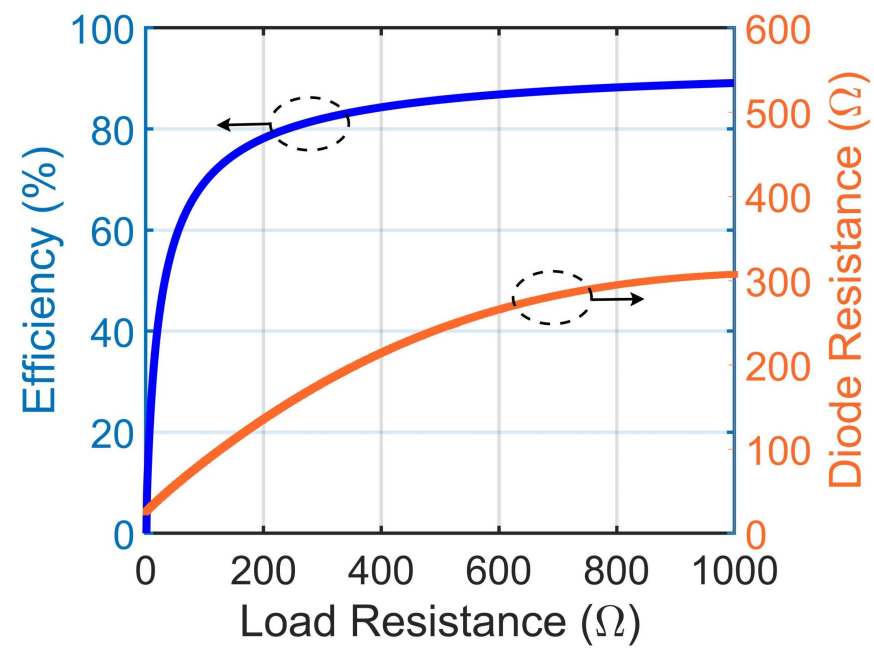

Figure 12. Numerical results of using the SMS 3922 Schottky diode model presented in [25]. The load resistance was varied from 0 to $1000 \Omega$ at $2 \mathrm{GHz}$.

The plot of the overall efficiency is presented in Figure 13. We can see that the overall efficiency is limited by the efficiency of the rectifier reaching an overall efficiency of $58 \%$. Such a drop in the efficiency can be attributed to the nonlinear behaviour of the diode generating harmonics, in addition to the losses introduced by the diode.

To highlight the novelty of the proposed dual polarized metasurface, Table 1 compares the novelty and advantages of the proposed harvesting array introduced in this study with a number of state of the art dual polarized harvesting arrays presented in the literature. While the studies in [14-21] provided high efficiency levels, they developed complicated or more complex structure by utilizing dual layers with vias and matching network structure which adds complexity to the design and fabrication of the metasurface array compared to the proposed metasurface array in this study. The developed metasurface array in this work achieves significantly higher radiation to AC efficiency (98\%) with single and simple layer structure avoiding the use of vias when compared with other developed rectenna arrays presented in the literature [14-21]. 


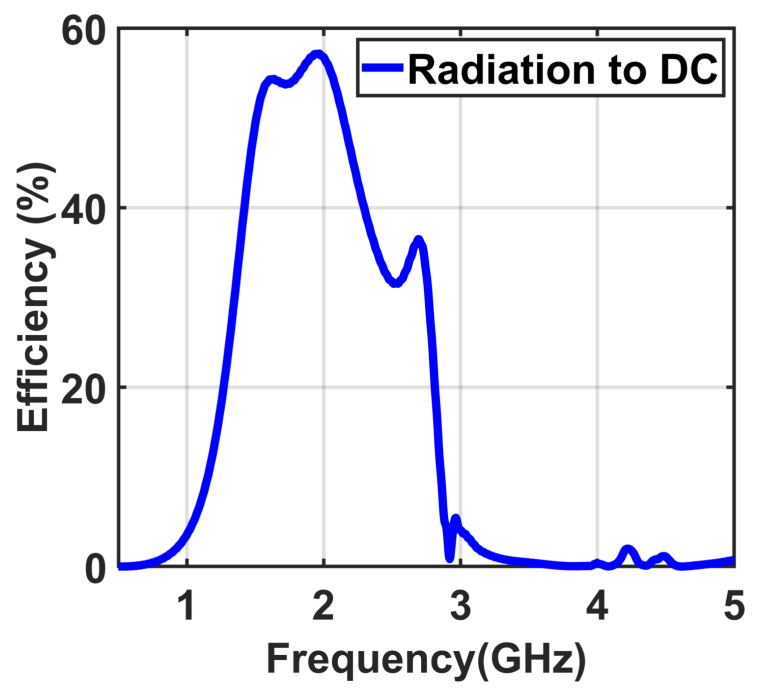

Figure 13. Numerical results showing the simulated radiation to dc efficiency.

Table 1. A comparative study of the proposed dual polarized rectenna array with various state of the art published papers.

\begin{tabular}{ccccccc}
\hline Reference & Footprint Area $\mathbf{( m m )}$ & Frequency $\mathbf{( G H z )}$ & Configuration Type & Using of Via & Number of Layers & Efficiency \\
\hline$[14]$ & $72 \times 72$ & 10 & Planar & Yes & Dual & $95.97 \%$ \\
{$[15]$} & $102.6 \times 102.6$ & 2.45 & Planar & Yes & Dual \\
{$[16]$} & $240 \times 240$ & 11.3 & Planar & Yes & Dual \\
{$[17]$} & $120 \times 120$ & 2.6 & Planar & Yes & Dual \\
{$[18]$} & $130.5 \times 130.5$ & 2.45 & Planar & Yes & Dual \\
{$[19]$} & $153 \times 153$ & 2.4 & Planar & Yes & Dual \\
{$[20]$} & $72 \times 83.5$ & 5.8 & Planar & Yes & Single \\
{$[21]$} & $139.3 \times 139.3$ & 3 & Planar & Yes & Single \\
{$[22]$} & $48 \times 48$ & 6.2 & Planar & Yes & Single \\
{$[23]$} & $70 \times 70$ & 5.8 & Planar & Yes & Single \\
This work & $200 \times 200$ & 1.7 & Planar & NO & Single \\
\hline
\end{tabular}

\section{Conclusions}

In this work, a simple and novel design of a dual polarized planar metasurface array for microwave energy harvesting is presented. The proposed independent polarized planer metasurface array is developed in a single-layer of $8 \times 8$ unit cells, which provides relatively high conversion efficiency. The simplicity and novelty of the developed polarization independent planer metasurface array is exhibited by using a single layer design and avoiding the use of dual layer structure with vias to connect the layers. In addition, the wide impedance response of the super unit cell eliminates the use of matching network which allows for placing diodes directly at the feed gap of the cells. Numerical results show that the proposed metasurface array is capable of converting the collected multi-polarized electromagnetic energy to AC power across the feed of the cells with efficiency reaching up to $98 \%$.

Author Contributions: The individual contributions of the authors can be summarized as follows: Conceptualization, M.A.A. and T.S.A.; methodology, M.A.A. and T.S.A; Simulation and software analysis, M.A.A. and T.S.A; investigation of the proposed idea, M.A.A. and T.S.A.; writing-original draft preparation, M.A.A. and T.S.A.; writing-review and editing, M.A.A. and T.S.A.; visualization, M.A.A. and T.S.A.; supervision, T.S.A.; project administration, T.S.A.; funding acquisition, T.S.A. All authors have read and agreed to the published version of the manuscript.

Funding: This research is funded by Deputyship for Research \& Innovation, through Ministry of Education in Saudi Arabia under project number 785 . 
Acknowledgments: The authors extend their appreciation to the Deputyship for Research \& Innovation, Ministry of Education in Saudi Arabia for funding this research work through the project number 785. The authors also acknowledge the support of the Deanship of Scientific Research at Prince Sattam bin Abdulaziz University, Alkharj, Saudi Arabia.

Conflicts of Interest: The authors declare no conflict of interest.

\section{References}

1. Amer, A.A.G.; Sapuan, S.Z.; Nasimuddin, N.; Alphones, A.; Zinal, N.B. A Comprehensive Review of Metasurface Structures Suitable for RF Energy Harvesting. IEEE Access 2020, 8, 76433-76452.

2. Awais, Q.; Jin, Y.; Chattha, H.T.; Jamil, M.; Qiang, H.; Khawaja, B.A. A compact rectenna system with high conversion efficiency for wireless energy harvesting. IEEE Access 2018, 6, 35857-35866.

3. Chen, Y.S.; Lai, F.P.; You, J.W. Analysis of antenna radiation characteristics using a hybrid ray tracing algorithm for indoor WiFi energy-harvesting rectennas. IEEE Access 2019, 7, 38833-38846.

4. Sun, H.; He, H.; Huang, J. Polarization-Insensitive Rectenna Arrays With Different Power Combining Strategies. IEEE Antennas Wirel. Propag. Lett. 2020, 19, 492-496.

5. Harouni, Z.; Cirio, L.; Osman, L.; Gharsallah, A.; Picon, O. A dual circularly polarized 2.45-GHz rectenna for wireless power transmission. IEEE Antennas Wirel. Propag. Lett. 2011, 10, 306-309.

6. Zhang, H.; Gao, S.P.; Wu, W.; Guo, Y.X. Uneven-to-even power distribution for maintaining high efficiency of dual-linearly polarized rectenna. IEEE Microw. Wirel. Components Lett. 2018, 28, 1119-1121.

7. Ou, J.H.; Pan, J.; Dong, S.W.; Zhang, X.Y. A Dual-Polarized Rectenna with High Efficiency at Low Input Power Density. In Proceedings of the 2020 14th European Conference on Antennas and Propagation (EuCAP), Copenhagen, Denmark, 15-20 March 2020; pp. 1-5.

8. Mattsson, M.; Kolitsidas, C.; Silver, O.B.G.; Dahlberg, O.; Jonsson, B. A high gain dual-polarised differential rectenna for RF energy harvesting. In Proceedings of the 2017 IEEE International Symposium on Antennas and Propagation \& USNC/URSI National Radio Science Meeting, San Diego, CA, USA, 9-14 July 2017; pp. 1609-1610.

9. Mahfoudi, H.; Takhedmit, H.; Tellache, M. Dual-band dual-polarized stacked rectenna for RF energy harvesting at 1.85 and $2.45 \mathrm{GHz}$. In Proceedings of the 12th European Conference on Antennas and Propagation (EuCAP 2018), London, UK, 9-13 April 2018.

10. Alavikia, B.; Almoneef, T.S.; Ramahi, O.M. Electromagnetic energy harvesting using complementary split-ring resonators. Appl. Phys. Lett. 2014, 104, 163903.

11. Ramahi, O.M.; Almoneef, T.S.; AlShareef, M.; Boybay, M.S. Metamaterial particles for electromagnetic energy harvesting. Appl. Phys. Lett. 2012, 101, 173903.

12. El Badawe, M.; Almoneef, T.S.; Ramahi, O.M. A metasurface for conversion of electromagnetic radiation to DC. AIP Adv. 2017, 7, 035112.

13. Almoneef, T.S.; Ramahi, O.M. Metamaterial electromagnetic energy harvester with near unity efficiency. Appl. Phys. Lett. 2015, 106, 153902.

14. Ayop, O.; Rahim, M.K.A.; Murad, N.A.; Samsuri, N.A. Double Layer Polarization Insensitive Metamaterial Absorber with Dual Resonances. In Theory and Applications of Applied Electromagnetics; Springer: New York, NY, USA, 2015; pp. 231-238.

15. Ghaderi, B.; Nayyeri, V.; Soleimani, M.; Ramahi, O.M. Pixelated metasurface for dual-band and multi-polarization electromagnetic energy harvesting. Sci. Rep. 2018, 8, 1-12.

16. Lim, D.; Lee, D.; Lim, S. Angle-and polarization-insensitive metamaterial absorber using via array. Sci. Rep. 2016, 6, 1-9.

17. El Badawe, M.; Ramahi, O. Polarization independent metasurface energy harvester. In Proceedings of the 2016 IEEE 17th Annual Wireless and Microwave Technology Conference (WAMICON), Clearwater Beach, FL, USA, 11-13 April 2016; pp. 1-3.

18. Costanzo, S.; Venneri, F. Polarization-Insensitive Fractal Metamaterial Surface for Energy Harvesting in IoT Applications. Electronics 2020, 9, 959.

19. Almoneef, T.S.; Erkmen, F.; Ramahi, O.M. Harvesting the energy of multi-polarized electromagnetic waves. Sci. Rep. 2017, 7, 1-14. 
20. Yu, F.; Yang, X.; Zhong, H.; Chu, C.; Gao, S. Polarization-insensitive wide-angle-reception metasurface with simplified structure for harvesting electromagnetic energy. Appl. Phys. Lett. 2018, 113, 123903.

21. Ashoor, A.Z.; Ramahi, O.M. Polarization-independent cross-dipole energy harvesting surface. IEEE Trans. Microw. Theory Tech. 2019, 67, 1130-1137.

22. Zhong, H.T.; Yang, X.X.; Song, X.T.; Guo, Z.Y.; Yu, F. Wideband metamaterial array with polarization-independent and wide incident angle for harvesting ambient electromagnetic energy and wireless power transfer. Appl. Phys. Lett. 2017, 111, 213902.

23. Yu, F.; Du, J.; Yang, X. Four-Band Polarization-Insensitive and Wide-Angle Metasurface with Simplified Structure for Harvesting Electromagnetic Energy. In Proceedings of the 2019 IEEE MTT-S International Wireless Symposium (IWS), Guangzhou, China, 19-22 May 2019; pp. 1-3.

24. CST. Computer Simulation and Technology. Available online: http:/ / www.CST.com (accessed on 5 July 2020).

25. McSpadden, J.O.; Fan, L.; Chang, K. Design and experiments of a high-conversion-efficiency 5.8-GHz rectenna. IEEE Trans. Microw. Theory Tech. 1998, 46, 2053-2060.

Publisher's Note: MDPI stays neutral with regard to jurisdictional claims in published maps and institutional affiliations.

(C) 2020 by the authors. Licensee MDPI, Basel, Switzerland. This article is an open access article distributed under the terms and conditions of the Creative Commons Attribution (CC BY) license (http:/ / creativecommons.org/licenses/by/4.0/). 